paired E-box sites or linked E-box and GATA binding sites. Interestingly, highly conserved motifs for other transcription factors, including Runx, Ets, PU.1, Gfil and Meis1, were frequently associated with Ldb1 occupancy. It is intriguing that Ldb1 binding at putative regulatory elements in HSCs is often linked with Runx, Ets and PU.1 occupancy. Recent global occupancy studies revealed that binding of $\mathrm{E} 2 \mathrm{~A}$ in pre-pro-B and pro- $\mathrm{B}$ cells is similarly primarily associated with Runx, Ets and PU.1 binding sites ${ }^{11}$. Thus, in HSCs and developing B-lineage cells, E-protein-containing complexes associate with similar collaborators at large subsets of putative regulatory elements. It will now be of interest to determine to what extent the binding patterns of E-box-binding proteins differ in a global sense between HSCs and maturing B cells. In other words, how is an HSC-specific gene expression program established from regulators that are not restricted in their expression patterns to HSCs? It is likely that this will involve the concerted activities of key regulators, rather than 'master regulators' that act by themselves, to induce lineagespecific gene-expression programs in HSCs as well as in differentiated hematopoietic cells.

In sum, the studies of $\mathrm{Li}$ et al. indicate that Ldb1 and its associated partners have an essential role as guardians of HSC integrity. Furthermore, this study has also brought together many of the factors previously linked to HSC maintenance in one common pathway. Building on this work, it should soon be possible to describe the maintenance of fetal and adult HSC pools in terms of global networks.

\section{COMPETING FINANCIAL INTERESTS}

The authors declare no competing financial interests.

1. Boiani, M. \& Schöler, H.R. Nat. Rev. Mol. Cell Biol. 6 872-884 (2005).

2. Souroullas, G.P. et al. Cell Stem Cell 4, 180-186 (2009).

3. Tsai, F.Y. et al. Nature 371, 221-226 (1994).

4. Semerad, C.L. et al. Proc. Natl. Acad. Sci. USA 106, 1930-1935 (2009).

5. Li, et al. Nat. Immunol. 12, 129-136 (2011).

6. Matthews, J.M. \& Visvader, J. EMBO Rep. 4, 1132-1137 (2003).

7. Wadman, I.A. et al. EMBO J. 16, 3145-3157 (1997)

8. Song, S.H., Hou, C. \& Dean, A. Mol. Cel/ 14, 810-822 (2007).

9. Soler, E. et al. Genes Dev. 24, 277-289 (2010).

10. Adolfsson, J. et al. Immunity 15, 659-669 (2001).

11. Lin, Y.C. et al. Nat. Immunol. 11, 635-643 (2010).

\title{
2 methylate or not 2 methylate: viral evasion of the type I interferon response
}

\author{
Adolfo García-Sastre \\ Recent work highlights the requirement for 2'-O-methylation of capped mRNA to prevent recognition by the antiviral \\ host response.
}

\begin{abstract}
The induction of antiviral responses in hosts and the evasion of these responses by viruses represent major selective forces for both types of organisms. As hosts evolved sophisticated mechanisms for viral recognition leading to antiviral immunity, viruses evolved counteracting mechanisms to evade recognition as well as the effects of the host antiviral response. This evolutionary battle has been ongoing almost since the origin of life, and has shaped many different aspects of the molecular processes in prokaryotic and eukaryotic organisms. Among the hallmarks of this battle were the acquisition by eukaryotic organisms of sensors of viral RNA products that trigger secretion of antiviral cytokines, such as type I interferon, and the subsequent acquisition by viruses of mechanisms to evade recognition by these sensors. The studies published by Züst et al. ${ }^{1}$ in this issue of Nature Immunology and by Daffis et al. ${ }^{2}$ in a recent issue of Nature reveal one of the surprising consequences of the ongoing coevolutionary forces that shape
\end{abstract}

Adolfo García-Sastre is at the Department of Microbiology, the Department of Medicine, Division of Infectious Diseases, and the Global Health and Emerging Pathogens Institute, Mount Sinai School of Medicine, New York, New York, USA. e-mail: adolfo.garcia-sastre@mssm.edu virus-host interactions and cellular and viral mRNA processing mechanisms.

Sensing of viral RNA products by the infected cell implies the challenge of distinguishing self versus non-self (viral) RNA. This challenge is overcome by the cellular cytoplasmic sensor RIG-I ${ }^{3}$. RIG-I recognizes $5^{\prime}$-triphosphatecontaining RNAs present in the genome of many RNA viruses ${ }^{4-7}$, but not in the cellular cytoplasmic RNAs. Self RNAs are modified to avoid the presence of this danger signal. RIG-I belongs to a small family of related proteins with RNA helicase activities. A second member of this family, Mda5, is also involved in the recognition of viral products, presumably viral RNA. Although it is known that several virus families, such as picornaviruses and coronaviruses, and long double-stranded RNAs, such as poly(I:C), are preferentially recognized by Mda5 (ref. 8), the actual pathogen-associated molecular pattern (PAMP) generated during viral infection that triggers Mda5 sensing has remained unknown. Züst et al. ${ }^{1}$ now show that mRNA lacking 2'-O-methylation at their $5^{\prime}$ cap structure induces production of type I interferon through Mda5 activation.

The cap structure of the cellular mRNAs is characterized by methylation at the $\mathrm{N} 7$ position of the $5^{\prime}$ guanosine residue. This modification is known to mediate optimal mRNA processing and translation. In addition, cellular mRNAs from higher eukaryotes are methylated at the ribose$2^{\prime}-O$ position of the second and sometimes third residue adjacent to the $5^{\prime}$ guanosine. The functional significance of $2^{\prime}$ - $O$-methylation has remained unclear. However, many virus families encode not only N7-methyltransferases but also $2^{\prime}$-O-methyltransferases that modify the 5 'end of their viral mRNAs, suggesting that this modification may be physiologically important. As shown by Züst $e t$ al. $.^{1}, 2^{\prime}$-O-methylation is critical to avoiding Mda5 recognition. Thus, mutant coronaviruses lacking 2'-O-methyltransferase activity induce type I interferon expression in an Mda5-dependent manner. In addition, these mutants are more sensitive to the antiviral action of type I interferon.

In a related study, Daffis et al. ${ }^{2}$ have characterized a mutant West Nile virus that is defective in 2'-O-methyltransferase activity. Like the mutant coronaviruses, the mutant West Nile virus was more sensitive to the antiviral action of interferon, and characterization of the mechanism responsible for this effect implicates the interferon-induced proteins IFIT. The IFIT proteins inhibit viral protein synthesis through translational arrest $^{9}$. Lack of 2'-O-methylation increased the sensitivity of West Nile virus to the antiviral action of the IFIT proteins. Thus, it appears that mRNAs devoid of 2'-O-methylation 
are being sensed by cellular Mda5 and IFIT proteins, resulting in interferon induction and translational inhibition. The West Nile virus mutant, in contrast to the coronavirus mutant, did not increase type I interferon induction. This might be explained by the presence of inhibitors of type I interferon production encoded by West Nile virus, as recently demonstrated for the closely related dengue virus ${ }^{10}$.

The picture that emerges from these two studies is the following (Fig. 1). As higher eukaryotes acquired the ability to $2^{\prime}$ - $O$-methylate their mRNAs, this opened the possibility of distinguishing self from non-self mRNA through new types of antiviral sensors, such as Mda5 and IFITs. This evolutionary advantage was counteracted by the viral acquisition of $2^{\prime}-O$ methyltransferases; coronaviruses, flaviviruses and poxviruses, among others, adopted this evasion strategy. However, this is not the only strategy used by viruses to counteract detection. Some viruses known to transcribe mRNA in the nucleus, such as the human immunodeficiency virus (HIV), use the host cell mRNA capping machinery. Other viruses, such as the influenza virus, steal the cap from the cellular mRNAs to be used as primers for initiating synthesis of their viral mRNA. Finally, picornaviruses avoid the use of a cap altogether. Instead, they use internal ribosomal entry sites, which allows cap-independent protein translation. In summary, it seems that mammalian viruses cannot afford to produce mRNA containing a cap structure lacking 2'-O-methylation.

The studies by Züst et al. and Duffis et al. open up new questions related to viral recognition by cellular sensors. Is non-2'-O-methylated mRNA directly recognized by $\mathrm{Mda} 5$, or are there other cellular proteins upstream of Mda5 that are required for this process? What is the molecular mechanism used by the IFIT proteins to preferentially mediate translational arrest of

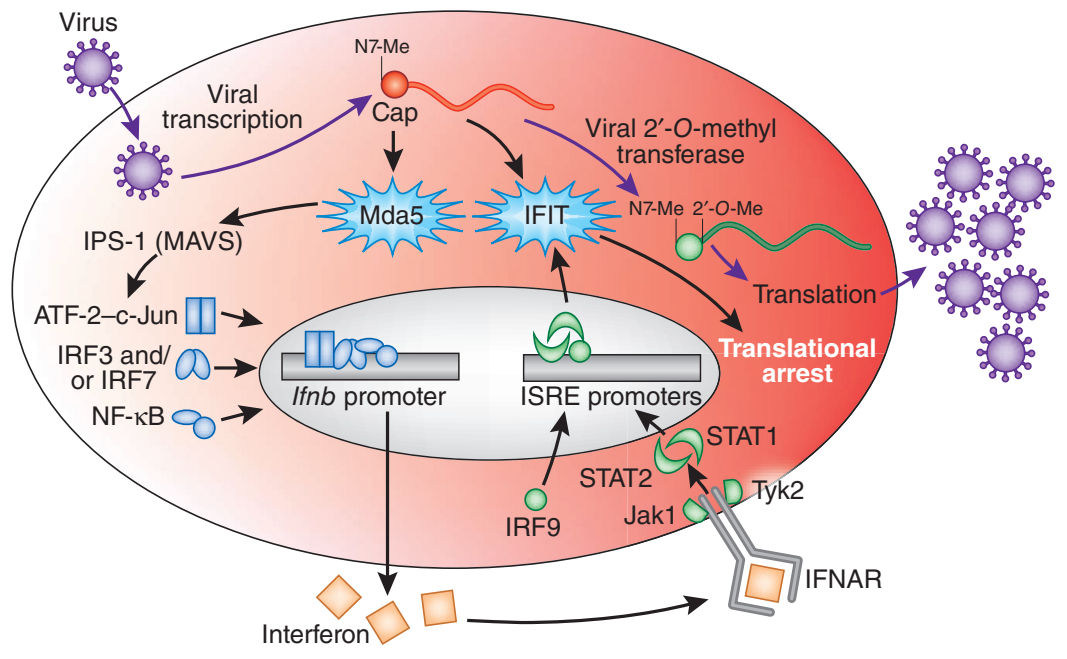

Figure 1 2'-O-methylation of viral mRNA avoids recognition by the cellular antiviral sensing machinery. In the case of viruses that replicate in the cytoplasm and do not have access to the cellular nuclear machinery responsible for mRNA capping, viral mRNA is synthesized by viral enzymes. Thus, many cytoplasmic viruses encode the functions required to cap and N7-methylate the viral mRNA. Although N7 methylation is sufficient to access the cellular translation machinery, 2'-O-methylation of the viral mRNA is also needed for it to avoid detection. In the absence of $2^{\prime}$-O-methylation, mRNAs induce the activation of the cellular sensor Mda5, which interacts with the downstream molecule IPS-1 (also called MAVS), resulting in activation of the latent transcription factors ATF-2, c-Jun, IRF3 and/or IRF7, and NF-KB, followed by type I interferon synthesis and secretion. Interferon binds to the interferon receptor IFNAR and initiates a signaling cascade involving the Jak1 and Tyk2 kinases and a transcription complex composed of STAT1, STAT2 and IRF9. This complex initiates the transcription of interferon-stimulated response element (ISRE) antiviral genes, such as the interferon-induced proteins with tricopeptide repeats (IFIT). IFITs inhibit cap-dependent translation, with a preference for mRNAs lacking 2'-O-methylation. Me, methyl; Ifnb, interferon- $\beta$ gene.

non-2'-O-methylated mRNA? Do other RNA modifications and cellular sensors participate in the distinction between self and non-self RNA? Can this information be used to design new antiviral inhibitors? Future studies are needed to elucidate these intriguing and interesting questions emerging from the millions of years of coevolution between viruses and hosts.

COMPETING FINANCIAL INTERESTS

The author declares no competing financial interests.
1. Züst, R. et al. Nat. Immunol. 12, 137-143 (2011). 2. Daffis, S. et al. Nature 468, 452-456 (2010).

3. Yoneyama, M. et al. Nat. Immunol. 5, 730-737 (2004).

4. Hornung, V. et al. Science 314, 994-997 (2006).

5. Cui, S. et al. Mol. Cell 29, 169-179 (2008).

6. Rehwinkel, J. et al. Cell 140, 397-408 (2010).

7. Baum, A., Sachidanandam, R. \& García-Sastre, A. Proc. Natl. Acad. Sci. USA 107, 16303-16308 (2010).

8. Kato, H. et al. J. Exp. Med. 205, 1601-1610 (2008)

9. Hui, D.J., Terenzi, F., Merrick, W.C. \& Sen, G.C. J. Biol. Chem. 280, 3433-3440 (2005).

10. Rodriguez-Madoz, J.R. et al. J. Virol. 84, 9760-9774 (2010).

\section{New friends for bone marrow plasma cells}

\section{Robert Brink}

\section{Long-lived plasma cells require a specialized bone marrow microenvironment in order to survive and produce antibody. Eosinophils make an important contribution to maintaining this survival niche.}

\footnotetext{
The bone marrow is one of the great cellular factories of the body, replenishing the stocks of nearly every cell type that makes up the

Robert Brink is in the Immunology Program,

Garvan Institute of Medical Research, Darlinghurst, New South Wales, Australia.

e-mail: r.brink@garvan.org.au
}

immune system. Among these are the B lymphocytes, whose task is to produce the secreted antibody proteins that bind to and eliminate invading foreign antigens. The development of $B$ cells within the bone marrow occurs within specialized microenvironments, or niches, that provide the nourishment required to keep precursor cells alive and committed to their job of producing the next generation of $\mathrm{B}$ cells ${ }^{1}$ (Fig. 1). After moving out of the bone marrow and into the periphery, mature B cells may be activated by foreign antigen, migrate into germinal centers and eventually emerge as plasmablasts encoding antibodies that can now bind foreign antigen with increased affinity (Fig. 1). These cells ultimately return to the bone marrow 